\title{
A Performance Model for the Link-Transport Layer Serving XTP in a High Speed Network
}

\author{
Meejeong Lee, Arne A. Nilsson and Rajesh I. Balay \\ Center for Communications and Signal Processing \\ Department of Electrical and Computer Engineering \\ North Carolina State University \\ Raleigh, NC 27695-7911
}

\begin{abstract}
How the traffic characteristics and the performance of a transport connection are affected at the link-transport layer is one of the important items to be studied to determine the end to end performance of a transport protocol. We model the link-transport layer that provides service to an XTP connection as a discrete time single server finite capacity queueing system at which two different arrival processes are allowed to merge, e.g., a Markov Modulated Bernoulli Process (MMBP) and a Bernoulli Process (BP). The MMBP models the bursty and correlated traffic from the designated XTP connection, and the BP models the external traffic, that is, the arrival of the packets from the rest of the coexisting connections at the link-transport layer. An exact analysis to obtain the queue length distributions, and the probability densities and the correlation coefficients of the interdeparture time distributions for the packets from the designated XTP connection is presented. Numerical examples are also investigated to show how the traffic characteristics of the designated XTP connection are affected by the amount of the external traffic, the degree of the burstiness and the correlation of the traffic source.
\end{abstract}

\section{INTRODUCTION}

The link-transport layer, the logical protocol layer immediately below the transport layer [1], may serve several transport connections at the same time. Thus, the arrival process to the link-transport layer is a superposition of multiple streams generated by different transport connections. How the characteristic of the traffic from a designated transport connection is influenced in the link-transport layer is an interesting problem and will be imperative in determining the end-to-end performance of a designated transport connection.

Heffs and Lucantoni have proposed an effective way to analyze a system with a superposed traffic consisting of multiple renewal processes[2]. By using their approach, however, only a performance prediction for the whole stream superposed together is obtained.

A per-stream analysis of a discrete-time system, where a GI-stream and a Bernoulli batch arrival process are superposed, is reported by Murata et al in the study of how the GIstream arrivals are affected in the system [3]. Ohba et al studied an extension of this system where they also considered a third arrival stream which represents the aggregate of many identical Interrupted Bernoulli Processes (IBP) [4]. However, neither of the analyses can account for the possible correlation in the designated arrival stream.

In this paper, a per-stream analysis of a discrete-time finite capacity queueing system with superposing traffic streams consisting of a correlated arrival stream and a Bernoulli arrival process is presented. The particular motivation for this study comes from the end-to-end performance analysis of the Xpress Transfer Protocol (XTP). The XTP, an emerging high performance protocol, is motivated by the needs of contemporary and future real-time, transactional, and multi-media systems[5]. A simulation study comparing the end-to-end performance of the different error control strategies allowed by the XTP definition is reported in [6]. We model the link-transport protocol layer, servicing several transport connections 
including an XTP connection, by the MMBP+BP/D(N)/1/K queueing system. The Markov Modulated Bernoulli Process (MMBP), a non-renewal arrival process, models the traffic from a designated XTP connection and the Bernoulli Process (BP) models the superposition of the traffic from the rest of the connections [7], [8], [9].

Due to the evolution in the network technology and the advent of new applications such as distributed system and multimedia services, the usual assumptions adopted for the analytical modeling of the link-transport protocol layer are no longer valid. Most of the past research on the performance of the packetized slotted communication networks has assumed that the input traffic follows a Bernoulli distribution, and that the processing delay at the communicating stations and the propagation delay are negligible compared to the transmission time [1]. However, the traffic sources that the XTP is supposed to support are mostly bursty and correlated, and thus the traffic from the XTP layer to the link-transport layer is no longer a smooth Bernoulli Process. Furthermore, the protocol processing and the propagation delay can be far larger than the transmission time in a high speed network. Predominately due to the use of fiber optic cables as a transmission medium, the error rates on communication channels have fallen significantly [10], and the dominant source of errors becomes packet loss due to buffer overflow. Therefore, the mathematical analysis of finite capacity queueing systems is very important for the understanding of the performance of communication systems.

In this paper, we model the traffic stream from a designated XTP connection by an MMBP model, and the link-transport protocol processor by a deterministic server with a finite capacity queue. The deterministic server in our queueing model requires multiple service slots indicating that the protocol processing may take longer time and sometimes much longer time than the transmission time.

The MMBP+BP/D(N)/1/K queue is analyzed to obtain the queue length (number of packets in the queue and in the server) distribution observed at arbitrary points in time and at the arrival instances of packets from the MMBP-stream. Furthermore, the probability distribution and the autocorrelation coefficient of the interdeparture time of the queueing system is obtained. The departure processes of queues are of special interest in the analysis of the queueing networks because it can be the arrival process to other queues, and the interdeparture time distribution and the autocorrelation coefficient are useful information in characterizing the departure process [11]. We also derive the waiting time distribution and the blocking probability for the MMBP-stream which models the traffic generated from the designated XTP connection.

This paper is organized as follows. In section 2, the queueing model $\mathrm{MMBP}+\mathrm{BP} / \mathrm{D}(\mathrm{N}) / 1 / \mathrm{K}$ is described and the probability density of the queue length distribution observed at arbitrary points in time is obtained by solving a multi-dimensional Markov chain. The probability density for the queue length distribution observed at the arrival instances of packets from the MMBP-stream is given in section 3. In section 4, the interdeparture time distribution for the traffic from the MMBP-stream is derived. The correlation coefficient of the interdeparture time for the MMBP-stream is obtained in section 5. In section 6, some numerical results obtained from our analysis for different traffic parameters are presented. Finally, conclusions are given in the last section.

\section{THE MMBP+BP/D(N)/1/K AT ARBITRARY POINTS IN TIME}

For the arrival process to our service system, we consider two kinds of traffic streams. The packets generated by the designated XTP connection arrive according to an MMBP [7], [8], and the packets from the other connections arrive in Bernoulli fashion. For the Bernoulli Process, the probability of an arrival in a slot time is denoted by $\beta$.

The two state MMBP is a doubly stochastic point process whose arrival phase process for each slot is governed by the two state irreducible Markov chain shown in Figure 1. If the MMBP is in state 1 (state 2) in the $n$th slot, it will remain in state 1 (state 2 ) in the $(n+1)$ st time 
slot with probability $p(q)$, or it will change to state 2 (state 1 ) with probability $1-p(1-q)$. Furthermore, if the $n$th time slot is in state $i,(i=1,2)$, arrivals occur according to a Bernoulli Process with probability $\alpha_{i}$.

An MMBP arrival process captures the notion of burstiness and correlation of the arrival stream. The burstiness of an arrival process is, in this paper, characterized by the squared coefficient of variation of interarrival time, $C^{2}$. The autocorrelation between successive interarrival times (i.e. with lag 1) is also an important measure that is considered. Given a certain offered load to the system the burstiness and the autocorrelation may change by varying in a careful way the values of $\mathrm{p}, \mathrm{q}, \alpha_{1}$ and $\alpha_{2}$.

The deterministic service time is assumed to be $N$ slots, and the state of the server is represented by the elapsed service time of the packet in service. Thus the state of the server ranges from 0 to $N$ with 0 identifying the idle state. We assume that the service for a packet may start at the earliest in the next slot following the slot with the arrival of the packet.

It is further assumed that arrivals can only occur at the beginning of each slot, and that departing packets leave the system at the end of each slot. The state of the MMBP changes only at the beginning of a slot just before arrivals occur, and the state of the server changes only at the end of a slot immediately after the potential departure time (see Figure 2). For simplicity, we assume that the MMBP-stream packet has priority over the Bernoulli Process packet in the sense that the MMBP-stream packet is served first if an MMBP-stream packet and a Bernoulli Process packet arrive in the same slot.

In order to obtain the steady state queue length distribution, we observe the system at the slot boundaries and generate the imbedded Markov chain [12]. In this Markov chain, there are $2(K+2)(N+1)$ states denoted by $(v, s, n)$ representing that the state of the MMBP is $\mathrm{v}$, the state of the server is $s$, and the number of packets in the system is $n . K$ is used to represent the buffer size, and thus at most $(K+1)$ packets are allowed in the system. There are only three events that can cause the Markov chain to change state: the MMBP state change, a packet arrival or a server state change. By solving the Global Balance Equations of the Markov chain, the queue length distribution of the MMBP+BP/D(N)/1/K queue is obtained.

\section{THE QUEUE LENGTH DISTRIBUTION OBSERVED AT THE ARRIVAL INSTANCES OF MMBP-STREAM PACKETS}

In this section, we obtain the queue length distribution observed by the arrivals from the MMBP-stream.This analysis will enable us to derive the waiting time distribution, the blocking probability, and the interdeparture time distribution for the MMBP-stream packets. The derivation of the interdeparture time distribution is given in detail in the following section.

The system state as defined above is represented by three parameters, the MMBP state, the state of the server, and the queue length. We observe the system state at arrival instances of packets from the MMBP-stream, and relate the distribution of the system state at the $(n+1)$ st observation point (the arrival instance of $(n+1)$ st MMBP-stream packet) to that of the previous observation point, i.e., the $n$th observation point [2], [3].

We define a random variable $C_{k}^{(n)}$ to represent the system state observed immediately before the potential arrival point in $k$ th slot following the $n$th MMBP-stream packet arrival given that the $(n+1)$ st MMBP-stream packet arrival does not occur in the preceding $k-1$ slots. $C_{k}^{(n)}$ is a 3-tuple variable, $C_{k}^{(n)}=\left(V_{k}^{(n)}, S_{k}^{(n)}, N_{k}^{(n)}\right)$, where $N_{k}^{(n)}$ and $S_{k}^{(n)}$ represent the queue length and the server state respectively observed immediately before the potential arrival point in the $k$ th slot following the $n$th arrival of a packet from the MMBP-stream, and $V_{k}^{(n)}$ indicates the MMBP state in which the $n$th MMBP-stream packet arrival occurred. Note that this variable doesn't 
change with $\mathrm{k}$. If $k=0, C_{0}^{(n)}$ is the system state seen by the $n$th MMBP-stream packet arrival. That is, $V_{0}^{(n)}, N_{0}^{(n)}$ and $S_{0}^{(n)}$ are the MMBP state,the queue length and the server state accordingly seen by the $n$th arrival of a packet from the MMBP-stream. The main objective in this section is to determine the probability distribution of $C_{0}^{(n)}$.

For this purpose, we further introduce supplemental random variables. $\bar{N}_{k}^{(n)}$ and $\bar{S}_{k}^{(n)}$ represent the queue length and the server state respectively observed immediately after the potential arrival point at the $k$ th slot following the $n$th arrival of a packet from the MMBPstream. $\bar{V}_{k}^{(n)}$ indicates the MMBP state in which the nth MMBP-stream packet arrival occurred. $\bar{C}_{k}^{(n)}$ is a 3-tuple variable, $\bar{C}_{k}^{(n)}=\left(\bar{V}_{k}^{(n)}, \bar{S}_{k}^{(n)}, \bar{N}_{k}^{(n)}\right)$. The order of occurrence of the random variable observation points in a slot is presented in Figure 3.

Immediately after an MMBP-stream packet arrival, there are $N_{0}^{(n)}+1+\mathrm{B}$ packets in the system, where $B$ is a random variable representing the number of arrivals from the Bernoulli Process stream. Hence, we have the relation

$$
\bar{N}_{0}^{(n)}=\min \left(N_{0}^{(n)}+1+B, K+1\right), \quad \text { where } \quad B=\left\{\begin{array}{ll}
1 & \text { with prob. } \quad \beta \\
0 & \text { with prob. } 1-\beta
\end{array} .\right.
$$

Next, we consider the relation between $\bar{N}_{0}^{(n)}$ and $N_{1}^{(n)}$. There are only three events that can happen between the two observation points of $\bar{N}_{0}^{(n)}$ and $N_{1}^{(n)}$ : a packet departure, a server state change, and the MMBP state change as shown in Figure 3. A departure occurs in a slot if and only if the state of the server before the potential server state change point is equal to $N$ in the given slot. Therefore, the relation is given by the equation

$$
N_{1}^{(n)}=\left\{\begin{array}{lc}
\bar{N}_{0}^{(n)} & 0 \leq S_{0}^{(n)}<N \\
\bar{N}_{0}^{(n)}-1 & S_{0}^{(n)}=N
\end{array} .\right.
$$

By similar reasoning, we have the following recurrence equations for $\mathrm{k}>0$.

$$
\begin{aligned}
& \bar{N}_{k}^{(n)}=\min \left(N_{k}^{(n)}+B, K+1\right), \\
& N_{k}^{(n)}=\left\{\begin{array}{ll}
\bar{N}_{k-1}^{(n)} & 0 \leq S_{k-1}^{(n)}<N \\
\bar{N}_{k-1}^{(n)}-1 & S_{k-1}^{(n)}=N
\end{array} .\right.
\end{aligned}
$$

By examining the order of events in a slot in Figure 3, the relations between $S_{k}^{(n)}$ and $\bar{S}_{k}^{(n)}$ for $k \geq 0$ can simply be derived as follows

$$
\begin{aligned}
& \bar{S}_{k}^{(n)}=S_{k}^{(n)},
\end{aligned}
$$

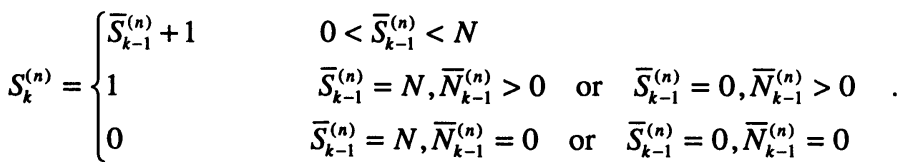


Next, we consider the probability density of the random variables. Let $C_{k}^{(n)}(v, i, j)$ and $\bar{C}_{k}^{(n)}(v, i, j)$ be the probability density functions of the random variables $C_{k}^{(n)}$ and $\bar{C}_{k}^{(n)}$ respectively, i.e., $C_{k}^{(n)}(v, i, j)=\operatorname{Prob}\left[V_{k}^{(n)}=v, S_{k}^{(n)}=i, N_{k}^{(n)}=j\right]$ and $\bar{C}_{k}^{(n)}(v, i, j)=\operatorname{Prob}$ $\left[\bar{V}_{k}^{(n)}=v, \bar{S}_{k}^{(n)}=i, \bar{N}_{k}^{(n)}=j\right]$.

have

Considering the relationship between the random variables as given by (1) - (6), we

$$
\begin{aligned}
& \bar{C}_{0}^{(n)}(v, i, j)=\left\{\begin{array}{ll}
C_{0}^{(n)}(v, i, j-1)(1-\beta)+C_{0}^{(n)}(v, i, j-2) \beta & 0 \leq j \leq K \\
C_{0}^{(n)}(v, i, K)+C_{0}^{(n)}(v, i, K-1) \beta+C_{0}^{(n)}(v, i, K+1) & j=K+1 \\
0 & j>K+1
\end{array},\right. \\
& v=0 \text { or } 1,0 \leq i \leq N, 0 \leq j \leq(K+1), \\
& \bar{C}_{k}^{(n)}(v, i, j)=\left\{\begin{array}{lc}
C_{k}^{(n)}(v, i, j)(1-\beta)+C_{k}^{(n)}(v, i, j-1) \beta & 0 \leq j \leq K \\
C_{k}^{(n)}(v, i, K) \beta+C_{k}^{(n)}(v, i, K+1) & j=K+1, \\
0 & j>K+1
\end{array}\right.
\end{aligned}
$$

$k \geq 1, v=0$ or $1,0 \leq i \leq N, 0 \leq j \leq(K+1)$,

$$
C_{k}^{(n)}(v, i, j)=\left\{\begin{array}{lc}
\bar{C}_{k-1}^{(n)}(v, 0,0)+\bar{C}_{k-1}^{(n)}(v, N, 1) & i=0, j=0 \\
\bar{C}_{k-1}^{(n)}(v, 0, j)+\bar{C}_{k-1}^{(n)}(v, N, j+1) & i=1, j>0 \\
\bar{C}_{k-1}^{(n)}(v, i-1, j) & 1<i \leq N, j>0 \\
0 & i=0, j>0 \text { or } 1 \leq i \leq N, j=0
\end{array},\right.
$$

$k \geq 1, v=0$ or $1,0 \leq i \leq N, 0 \leq j \leq(K+1)$.

When a new packet from the MMBP-stream arrives in the $k$ th slot following the last arrival, the new packet finds the queue length $N_{k}^{(n)}$ and the state of the server $S_{k}^{(n)}$ upon its arrival. Thus, the distribution of the system state seen by the $(n+1)$ st MMBP-stream packet arrival, by using the distribution of the system state in the $k$ th slot following the $n$th MMBPstream packet and the probability distribution that the $(n+1)$ st MMBP-stream packet arrival occurs in the $k$ th slot, can be obtained.

Since the MMBP is not a renewal process but a Markov renewal process, the distribution of the time interval from $n$th arrival to $(n+1)$ st arrival is not exponentially distributed but depends on the MMBP state at the time of the $n$th arrival. Therefore, we must use the conditional interarrival time distribution $a_{v}\left(v^{\prime}, k\right)$ (Prob[interarrival time $=k$ slots, state of the MMBP $=v^{\prime}$ | state of the MMBP for the previous arrival $\left.\left.v\right]\right)$, instead of $a(k)$ (Prob[interarrival time $=k$ slots]). The probability density $C_{0}^{(n+1)}(v, i, j)$ can be written as a function of $C_{k}^{(n)}(v, i, j)$ as follows

$$
C_{0}^{(n+1)}\left(v^{\prime}, i, j\right)=\sum_{k=1}^{\infty} a_{v}\left(v^{\prime}, k\right) C_{k}^{(n)}(v, i, j)
$$


The steady-state probability that there are $j$ packets in the queue, the state of MMBP is $v$, and the state of the server is $i$ upon a packet arrival from the MMBP-stream is given by

$$
C_{0}(v, i, j)=\lim _{n \rightarrow \infty} C_{0}^{(n)}(v, i, j), \quad v=0 \text { or } 1, \quad 0 \leq i \leq N, \quad 0 \leq j \leq(K+1) .
$$

Using $C_{0}(v, i, j)$, the joint probability density of the MMBP state, the queue length and the server state observed by a packet arrival from the MMBP-stream, the probability density of the queue length observed at the arrival instances of MMBP-stream packets can be simply obtained as follows

$\operatorname{Prob}[q u e u e$ length $=j$ la packet arrival from $M M B P$ stream $]=\sum_{v=0}^{1} \sum_{i=0}^{N} C_{0}(v, i, j)$.

The waiting time distribution and the blocking probability for the MMBP-stream packets are also readily obtained using $C_{0}(v, i, j)$.The waiting time for an MMBP-stream packet is the time to serve all the packets found in the queue and in the server upon the arrival of the given MMBP-stream packet. Since we assume that the MMBP-stream packet has priority over the Bernoulli Process stream packet when an MMBP-stream packet and a Bernoulli Process stream packet arrive at the same slot, the waiting time of the MMBP-stream packet is not affected by the possible arrival from Bernoulli Process stream in the same slot. Given that the state of the server was $i(>0)$ and that the number of packets in the system was $j$ upon the given MMBPstream packet arrival, the packet in the server requires $N-i+1$ time slots before its departure and the packets in the queue require $(j-1) \times N$ time slots to be served. Thus the total waiting time is $(N-i+1)+(j-1) \times N$ slots, given that the MMBP arrival sees that the server state was $i(>0)$ and the queue length was $j$ upon its arrival. Thus, the probability density of the waiting time for the MMBP-stream packet is given as a function of $C_{0}(v, i, j)$ as follows

$$
d(k)=\left\{\begin{array}{ll}
\sum_{v=0}^{1} \sum_{i=1}^{N} \sum_{j=1}^{K+1} C_{0}(v, i, j) \delta(k, j \times N-i+1), & k>0 \\
\sum_{v=0}^{1} C_{0}(v, 0,0) & k=0
\end{array},\right.
$$

where $\delta(a, b)$ is the Kronecker delta function.

The blocking probability for the MMBP-stream is the probability that an MMBP-stream arrival sees $(K+1)$ packets in the queue, and is given by $\sum_{v=0}^{1} \sum_{i=1}^{N} C_{0}(v, i, K+1)$.

\section{THE INTERDEPARTURE TIME DISTRIBUTION FOR THE MMBP-STREAM PACKETS}

In this section, we derive the interdeparture time distribution for packets from the MMBP-stream by using $C_{0}(v, i, j)$, the probability density of the system state observed at the arrival instances of the MMBP-stream packets, obtained in the previous section. 
For this purpose, we define the two-tuple random variables, $C_{k, s, q}^{(n)}=\left(N_{k, s, q}, S_{k, s, q}\right)$ and $\bar{C}_{k, s, q}^{(n)}=\left(\bar{N}_{k, s, q}, \bar{S}_{k, s, q}\right) . \quad N_{k, s, q}$, and $S_{k, s, q}$ represent the queue length and the server state accordingly observed immediately before the potential arrival point at the $k$ th slot following an arrival of a packet from the MMBP-stream, and $\bar{N}_{k, s . q}$, and $\bar{S}_{k, s, q}$ represent the queue length and the server state respectively observed immediately after the potential arrival point at the $k$ th slot following an arrival of a packet from the MMBP-stream, given that the state of the server was $s$ and the queue length was $q$ upon the arrival of the given MMBP-stream packet, and the next MMBP-stream packet arrival does not occur in the preceding $k-1$ slots.

By an argument similar to the one used in deriving (1) - (6), the relationships between the above defined random variables are as follows

$$
\begin{aligned}
& \bar{N}_{1, s, q}=\min (q+1+B, K+1), \\
& N_{k, s, q}=\left\{\begin{array}{ll}
\bar{N}_{k . s . q} & 0 \leq S_{k-1, s, q}<N \\
\bar{N}_{k . s . q}-1 & S_{k-1, s, q}=N
\end{array}\right. \text {, } \\
& k \geq 1 \text {, } \\
& \bar{N}_{k . s . q}=\min (q+B, K+1) \text {, } \\
& k>1 \text {, } \\
& \bar{S}_{k, s, q}=S_{k-1, s, q} \text {, } \\
& S_{k, s, q}= \begin{cases}\bar{S}_{k, s, q}+1 & 0<\bar{S}_{k, s, q}<N \\
1 & \bar{S}_{k, s, q}=N, \bar{N}_{k . s . q}>0 \text { or } \bar{S}_{k, s, q}=0, \bar{N}_{k, s . q}>0 \\
0 & \bar{S}_{k, s, q}=N, \bar{N}_{k . s . q}=0 \text { or } \bar{S}_{k, s, q}=0, \bar{N}_{k . s . q}=0\end{cases}
\end{aligned}
$$

The corresponding joint probability densities are denoted as $C_{k, s, q}(i, j)=$ $\operatorname{Prob}\left[S_{k, s, q}=i, N_{k . s . q}=j\right]$ and $\bar{C}_{k, s, q}(i, j)=\operatorname{Prob}\left[\bar{S}_{k, s, q}=i, \bar{N}_{k, s . q}=j\right]$ and are determined through the following equations.

$$
\begin{aligned}
& \bar{C}_{1, s, 0}(i, j)=\left\{\begin{array}{lc}
\beta & s=0, j=2, i=0 \\
1-\beta & s=0, j=1, i=0 \\
0 & \text { otherwise }
\end{array}\right. \\
& 0 \leq s, i \leq N, \quad 0 \leq j \leq(K+1), \\
& \bar{C}_{1, s, q}(i, j)=\left\{\begin{array}{lc}
\beta & s \neq 0, j=q+2, i=s \\
1-\beta & s \neq 0, j=q+1, i=s \\
0 & \text { otherwise }
\end{array}\right. \\
& 0 \leq s, i \leq N, \quad 0<q<K, \quad 0 \leq j \leq(K+1),
\end{aligned}
$$




$$
\begin{aligned}
& \bar{C}_{1, s, q}(i, j)= \begin{cases}1 & s \neq 0, i=s, j=K+1 \\
0 & \text { otherwise }\end{cases} \\
& 0 \leq s, i \leq N, \quad q=K, K+1, \quad 0 \leq j \leq(K+1),
\end{aligned}
$$

$$
\bar{C}_{k, s, q}(i, j)= \begin{cases}C_{k-1, s, q}(i, j)(1-\beta)+C_{k-1, s, q}(i, j-1) \beta & 0 \leq j \leq K \\ C_{k-1, s, q}(i, K) \beta+C_{k-1, s, q}(i, K+1) & j=K+1 \\ 0 & j>K+1\end{cases}
$$

$k>1, \quad 0 \leq s, i \leq N, \quad 0 \leq q, j \leq(K+1)$,

$$
C_{k, s, q}(i, j)= \begin{cases}\bar{C}_{k, s, q}(0,0)+\bar{C}_{k, s, q}(N, 1) & i=0, j=0 \\ \bar{C}_{k, s, q}(0, j)+\bar{C}_{k, s, q}(N, j+1) & i=1, j>0 \\ \bar{C}_{k, s, q}(i-1, j) & 1<i \leq N, j>0 \\ 0 & i=0, j>0 \text { or } 1 \leq i \leq N, j=0\end{cases}
$$

$k \geq 1, \quad 0 \leq s, i \leq N, \quad 0 \leq q, j \leq(K+1)$.

Now, we consider the interdeparture time between two MMBP-stream packets. The system time of a packet from the MMBP-stream is defined as the length of the time interval starting from the slot when the arrival of the given packet occurs and ending at the slot when the given packet departs the system. Let $w(s, q)$ denote the system time of a packet from the MMBP-stream given that the queue length was $q$ and the server state was $s$ upon its arrival. Then

$$
w(s, q)= \begin{cases}(q-1) N+(N-s+1)+N & q>0 \\ N+1 & q=0\end{cases}
$$

Using $w(s, q)$, the random variable $I D_{k, s, q}$ denoting the interdeparture time between two consecutively arriving packets from the MMBP-stream when the interarrival time between two packets is $k$, and the server state and the queue length upon the arrival of the first packet of the two are $s$ and $q$ respectively, is given by

$$
I D_{k, s, q}=k+w\left(S_{k, s, q}, N_{k, s, q}\right)-w(s, q) .
$$

From (23) and (24), $i d_{k, s, q}(j)$, the probability density of the random variable $I D_{k, s, q}$, is obtained as

$$
i d_{k, s, q}(j)= \begin{cases}\sum_{i=1}^{N}\left\{\begin{array}{l}
C_{k, s, q}(i,(j-k+i+q N-s) / N) \\
+C_{k, s, q}(0,0) \delta(j, k-q N+s)
\end{array}\right\} & q>0 \\
\sum_{i=1}^{N}\left\{\begin{array}{l}
C_{k, s, q}(i,(j-k+i) / N) \\
+C_{k, 0,0}(0,0) \delta(j, k)
\end{array}\right\} & q=0\end{cases}
$$

Finally, we remove the conditions from the above equations to obtain the probability density of the interdeparture time for the MMBP-stream as follows 


$$
i d(j)=\sum_{k=1}^{\infty} \sum_{s=0}^{N} \sum_{q=0}^{K+1} \sum_{v=0}^{1} a_{v}(k) C_{0}(v, s, q) i d_{k, s, q}(j), \quad a_{v}(k)=\sum_{v^{\prime}=0}^{1} a_{v}\left(v^{\prime}, k\right)
$$

\section{THE AUTOCORRELATION OF THE INTERDEPARTURE TIME FOR THE MMBP-STREAM PACKETS}

In this section, the autocorrelation coefficient of the interdeparture time for the MMBPstream packets is derived [7]. We define two random variables: $T_{n}$, the interdeparture time between the (n-1)st and the nth packets; $T_{n,(v, s, q)}$, the interdeparture time between the $(n-1)$ st and the $n$th packets given that the state of the system upon the $n$th packet arrival from the MMBPstream is $(v, s, q)$.

The autocorrelation coefficient of the interdeparture time of the MMBP-stream with lag 1 is given by

$$
\varphi_{1}=\frac{\operatorname{Cov}\left(T_{n-1} T_{n}\right)}{\operatorname{Var}\left(T_{n}\right)}=\frac{E\left(T_{n-1} T_{n}\right)-E\left(T_{n-1}\right) E\left(T_{n}\right)}{E\left(T_{n}^{2}\right)-E^{2}\left(T_{n}\right)} .
$$

We can easily determine $E\left(T_{n}\right)\left(=E\left(T_{n-1}\right)\right)$ by using the probability density of the interdeparture time distribution for the MMBP-stream derived in the previous section. To obtain the term $E\left(T_{n-1} T_{n}\right)$, we again introduce a number of z-transforms. Let

$$
\begin{aligned}
& A_{(v, s, q)\left(v^{\prime}, s^{\prime}, q^{\prime}\right)}(z) \equiv E\left[z^{T_{n,\left(v^{\prime}, s^{\prime}, q^{\prime}\right)}} \mid C_{0}^{(n-1)}=(v, s, q)\right], \\
& B_{(v, s, q)}(z) \equiv E\left[z^{T_{n}} \mid C_{0}^{(n-1)}=(v, s, q)\right], \\
& \text { and } C_{(v, s, q)}\left(z_{1}, z_{2}\right) \equiv E\left[z_{1}^{T_{n-1}} z_{2}^{T_{n}} \mid C_{0}^{(n-1)}=(v, s, q)\right] .
\end{aligned}
$$

To derive $A_{(v, s, q)\left(v^{\prime}, s^{\prime}, q^{\prime}\right)}(z)$, we define $t_{(v, s, q)\left(v^{\prime}, s^{\prime}, q^{\prime}\right)}$ as the time interval beginning from a particular slot at which a packet, whose arrival occurred when the state of the system was $(v, s, q)$, from the MMBP-stream departs and ending at a slot when the next MMBP-stream packet, upon whose arrival the state of the system was $\left(v^{\prime}, s^{\prime}, q^{\prime}\right)$, departure occurs. $t_{(v, s, q)\left(v^{\prime}, s^{\prime}, q^{\prime}\right)}=j$ with probability $p_{(v, s, q)\left(v^{\prime}, s^{\prime}, q^{\prime}\right)}(j)=$ prob[interdeparture time of the $n$th and the $(n+1)$ st MMBP-stream packets $=j$, state of the system upon the arrival of the $(n+1)$ st MMBPstream packet $=\left(v^{\prime}, s^{\prime}, q^{\prime}\right) \mid$ state of the system upon the arrival of the nth MMBP-stream packet $=(v, s, q)]$. Therefore,

$$
A_{(v, s, q)\left(v^{\prime}, s^{\prime}, q^{\prime}\right)}(z)=\sum_{j=1}^{\infty} p_{(v, s, q)\left(v^{\prime}, s^{\prime}, q^{\prime}\right)}(j) z^{j}
$$

$p_{(v, s, q)\left(v^{\prime}, s^{\prime}, q^{\prime}\right)}(j)$ can be obtained by using the conditional interarrival time distribution $a_{v}\left(v^{\prime}, k\right)$ and the probability distribution of the system state observed at the arrival instances of the MMBP-stream packets $C_{0}(v, s, q)$ 


$$
p_{(v, s, q)\left(v^{\prime}, s^{\prime}, q^{\prime}\right)}(j)=\sum_{k=1}^{\infty} a_{v}\left(v^{\prime}, k\right) C_{0}(v, s, q) \delta\left(j,\left\{k+w\left(s^{\prime}, q^{\prime}\right)-w(s, q)\right\}\right) .
$$

By using (28), we obtain

$$
\begin{aligned}
& B_{(v, s, q)}(z)=\sum_{v^{\prime}=0}^{1} \sum_{s^{\prime}=0}^{N} \sum_{q^{\prime}=0}^{K+1} A_{(v, s, q)\left(v^{\prime}, s^{\prime}, q^{\prime}\right)}(z) \\
& C_{(v, s, q)}\left(z_{1}, z_{2}\right)=\sum_{v^{\prime}=0}^{1} \sum_{s^{\prime}=0}^{N} \sum_{q^{\prime}=0}^{K+1} A_{(v, s, q)\left(v^{\prime}, s^{\prime}, q^{\prime}\right)}\left(z_{1}\right) B_{\left(v^{\prime}, s^{\prime}, q^{\prime}\right)}\left(z_{2}\right)
\end{aligned}
$$

Further, we denote the matrix of $A_{(v, s, q)\left(v^{\prime}, s^{\prime}, q^{\prime}\right)}(z)$, the vector of $B_{(v, s, q)}(z)$, and the vector of $C_{(v, s, q)}\left(z_{1}, z_{2}\right)$ by $A(z), B(z)$, and $C\left(z_{1}, z_{2}\right)$ respectively. Equation (31) is then rewritten in matrix form as follows

$$
C\left(z_{1}, z_{2}\right)=A\left(z_{1}\right) B\left(z_{2}\right) .
$$

By the definition of $C_{(v, s, q)}\left(z_{1}, z_{2}\right)$ in (28) and equation (31), it is obvious that

$$
E\left(z_{1}^{T_{n-1}} z_{2}^{T_{n}}\right)=C_{0} C\left(z_{1}, z_{2}\right)=C_{0} A\left(z_{1}\right) B\left(z_{2}\right),
$$

where $C_{0}$ is the probability vector of the probability density $C_{0}(v, s, q)$.

Finally, we can readily derive $E\left(T_{n-1} T_{n}\right)$ from $E\left(z_{1}^{T_{n-1}} z_{2}^{T_{n}}\right)$ as

$$
E\left(T_{n-1} T_{n}\right)=\left.C_{0} \frac{d A\left(z_{1}\right)}{d z_{1}} \frac{d B\left(z_{2}\right)}{d z_{2}}\right|_{z_{1}=1, z_{2}=1}
$$

, and the autocorrelation coefficient of the interdeparture time for the MMBP-stream is obtained by substituting $E\left(T_{n-1} T_{n}\right)$ in equation (27).

\section{NUMERICAL RESULTS}

In this section, numerical examples are presented by employing the analytical approach presented above. For the numerical computation to be tractable, we approximate the interarrival time and the interdeparture time distribution between packets such that the maximum time is finite. We will investigate how the traffic characteristics of the MMBP-stream are influenced by the amount of BP-stream traffic, the degree of the burstiness and the correlation of the MMBPstream.

The parameters assumed for the arrival process and the system are given in each figure. $\lambda, \mathrm{CC}, \mathrm{C}^{2}, \beta, \mathrm{K}$, and $\mathrm{N}$ represent the average arrival rate of an arrival for the MMBP-stream, the autocorrelation coefficient of the MMBP-stream, the $\mathrm{C}^{2}$ value of the MMBP-stream, the arrival rate of the BP-stream, the buffer capacity of the system, and the deterministic service time respectively.

Figures 4 and 5 show how the probability densities of the interdeparture times of the MMBP-stream change as the BP-stream traffic increases. For comparison purpose, we also include the probability density for the interarrival time distributions of the MMBP-stream. 
In Figure 4, we observe that the probability densities are less peeked as the arrival rate of the BP-stream increases $(\beta=0.1,0.3,0.5)$. That is, the probability of interdeparture time equal to 1 gets smaller and that of the interdeparture time equal to 2 gets larger as the amount of the BP-stream increases. This is because the packets from the BP-stream tend to interleave the continuously arriving packets from the MMBP-stream.

In Figure 5, however, we observe the opposite phenomina, i.e., the probability densities of interdeparture time gets more peeked as the amount of the traffic from the BPstream increases $(\beta=0.5,0.9,1.0)$. When $\beta$ is equal to 1.0 , the interdeparture time probability density nearly overlaps the interarrival time probability density. As the value of $\beta$ increases the buffer gets full more frequently and large amount of BP-stream packets are lost. This results in reducing the effect of the BP-stream to the performance of the MMBP-stream, and thus the probability density of the interdeparture time becomes similar to the original interarrival time distribution of the MMBP-stream.

Next, we see the autocorrelation coefficient with lag 1 and the $C^{2}$ of the interdeparture time of the MMBP-stream packets dependent on the amount of the traffic from the BP-stream in Figures 6 and 7. We observe that the correlation of the interdeparture time becomes larger, and the $\mathrm{C}^{2}$ of the interdeparture time gets smaller as the arrival rate of the BP-stream increases. The change is more rapid when $\lambda=0.6$ than when $\lambda=0.2$. However, the correlation starts to decrease and the $\mathrm{C}^{2}$ starts to increase as the arrival rate of the BP-stream increases to exceed a certain limit due to the BP-stream packet loss as explained above. When $\lambda=0.6$, the value of this limit is smaller. In the extreme case, when $\beta=1.0$, the correlation and the $C^{2}$ of the departure process approach to those of the arrival process of the MMBP-stream.

Figures 8 shows the mean waiting time for a packet from the MMBP-stream as a function of the amount of traffic from the BP-stream. Since the deterministic service time is set to 1 , there is no waiting for MMBP-stream packets if $\beta$ is 0.0 , i.e., no BP-stream traffic. Once the system becomes full, the system stays in the full state when $\beta$ is 1.0. Thus, the waiting time of an MMBP-stream packet when $\beta$ is 1.0 is equal to the buffer capacity, $K=8$. We observe that the mean waiting time when $\lambda=0.6$ grows more rapidly than that $\lambda=0.2$ as $\beta$ increases from 0.3 to 0.4 , see in Figure 8. This is because the buffer is filled up faster when the total load of the system is larger, that is, when $\lambda$ is larger. Since we have a finite capacity system, the amount of mean waiting time increase is reduced as the total load increases to exceed a certain limit due to packet loss.

Figure 9 shows the blocking probability for the MMBP-stream packets. Similar to the mean waiting time, the blocking probability increases rapidly as $\beta$ increases, and as the load of the system exceeds a certain limit the amount of increase is reduced.

The mean waiting time and the probability of blocking for the MMBP-stream packets dependent on the correlation and the burstiness of the MMBP-stream are shown in Figures 10 13. The mean waiting time and the blocking probability for the MMBP-stream increases in a logarithmic fashion with increase in burstiness and show an exponential type increase with increase in autocorrelation.

Figures 14 and 15 show the queue length distribution at arbitrary points in time. Figures 16 and 17 show the queue length distribution at MMBP-stream packet arrival instances dependent on the autocorrelation and the burstiness of the MMBP-stream. We observe that the queue length distributions at arbitrary point in time are less sensitive to the changes of MMBPstream characteristics. 


\section{CONCLUSION}

In this paper, we studied a queueing model which is particularly motivated by the study of the link-transport layer servicing several transport connections including an XTP connection in a high speed network. We have analyzed a discrete time finite capacity queueing system with a deterministic service requirement of multiple time slots. For the arrival processes, two kinds of traffic streams, MMBP and Bernoulli Process, are considered.

The queue length distribution of MMBP+BP/D(N)/1/K queue at arbitrary points in time using a multi-dimensional Markov chain analysis is obtained first. The queue length distribution observed at the MMBP-stream packet arrival instances, and the waiting time distribution and the blocking probability for the MMBP-stream are then obtained to investigate how the MMBPstream is affected in the queue. The probability density and the autocorrelation coefficient of the interdeparture time distribution for the MMBP-stream, which are important in the analysis of network of queues, are also derived.

The numerical examples show that the traffic characteristics and the performance for the designated transport connection are affected by the external traffic load, the degree of burstiness and correlation of the traffic source.

\section{REFERENCES}

[1] W. Stallings, Data and Computer Communications, Macmillan Publishing Company, Macmillan, Inc., NJ, pp134-147, 1991.

[2] H.Heffes and D. M. Lucantoni, "A Markov modulated characteristics of packetized voice and data traffic and related multiplexer performance," IEEE J. on Select. Areas Commun., vol. SAC-4, no. 6, pp. 856-868, Sept. 1986.

[3] Masayuki Murata, Yuji Oie, and Tatsuya Suda, "Analysis of a Discrete-Time Singleserver Queue with Bursty Inputs for Traffic Control in ATM Networks," IEEE J. on Select. Areas Commun., vol. 8, no. 3, April 1990.

[4] Yoshihiro Ohba, Masayuki Murata, and Hideo Miyahara, "Analysis of Interdeparture Processes for bursty Traffic in ATM Networks," IEEE J. on Select. Areas Commun., vol. 9, no. 3, April 1991.

[5] XTP Protocol Definition Revision 3.6, PEI, Santa Barbara, CA, 1992.

[6] Arne A. Nilsson and Meejeong Lee, "A Performance Study of the XTP Error Control," Proc. 4th IFIP conference on High Performance Networking, Liege, 1992.

[7] W. Fischer and K. Meier-Hellstern, "The MMPP Cookbook," draft, Oct. 1990.

[8] U. Briem, T. H. Theimer and H. Kroner, "A General Discrete-Time Queueing Model: Analysis and Applications," TELETRAFFIC AND DATATRAFFIC in a period of Change, ITC-13, Elsevier Science Publishers B.V. (North-Holland) IAC, 1991.

[9] J.R. Louvion, P. Boyer, and A. Gravey, "A Discrete-Time Single Server Queue with Bernoulli Arrivals and Constant Service Time," Proc. 12th International Teletraffic Congress, Torino, 1988.

[10] I. Jacobs, "Design considerations for long-haul lightwave systems," IEEE J. Select. Areas Commun., vol. SAC-4, pp. 1389-1395.

[11] Dooyeong Park, Henry G. Perros, and Hideaki Yamashita, "Approximate Analysis of Discrete-time Tandem Queueing Networks with Bursty and Correlated Input Traffic and Customer Loss," Technical Report, Department of Computer Science, North Carolina State University, Raleigh, NC, 1992.

[12] L. Kleinrock, Queueing System, Vol. I, John Wiley \& Sons, Inc., New York, NY, PP. 126-130, 1975. 


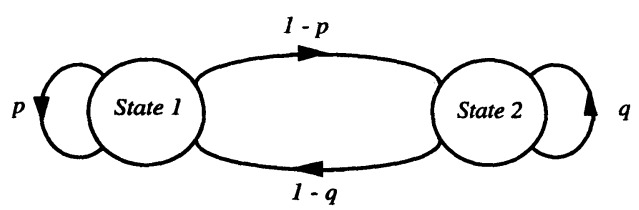

Figure 1 The Markov chain of a two-state MMBP.

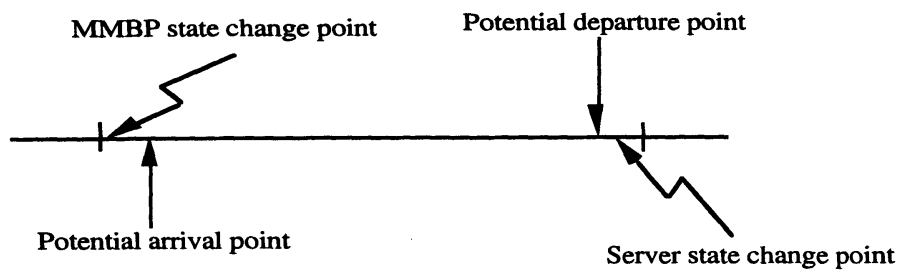

Figure 2 Order of events in a slot.

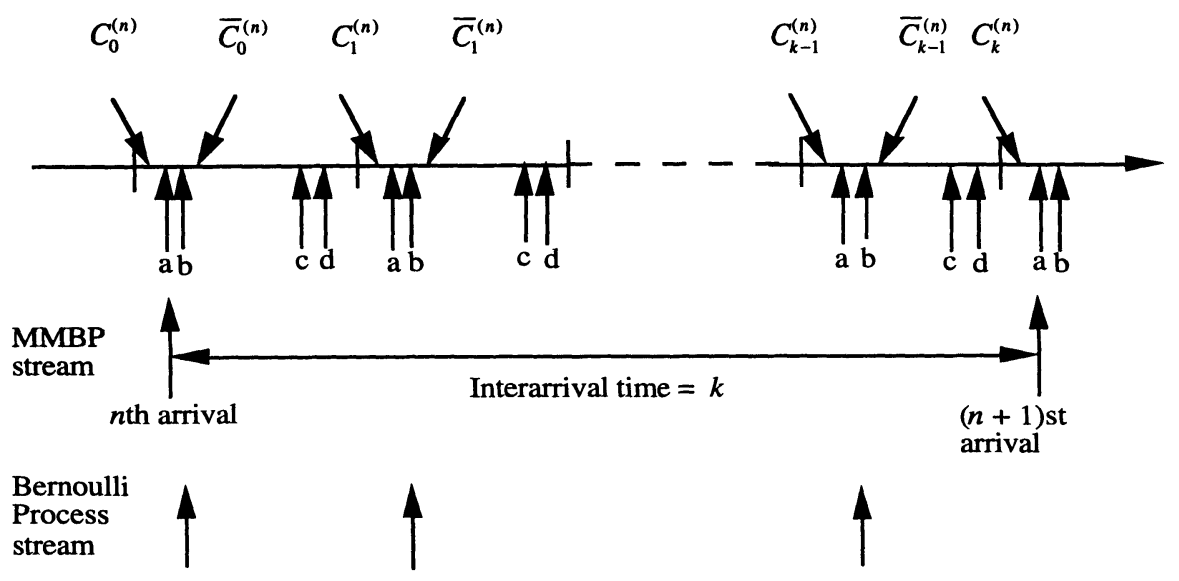
a: MMBP state change point
b: potential arrival point
c: potential departure point
d: server state change point

Figure 3 The order of occurrence of the random variable observation points. 


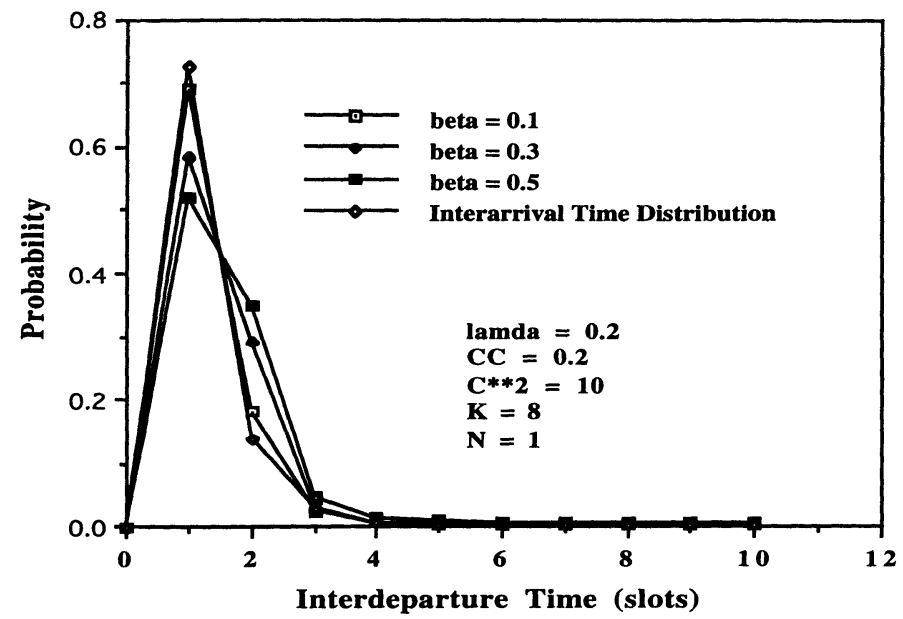

Figure 4 Interdeparture time distribution for the MMBP-stream w.r.t. the amount of the BP stream ( $\beta=0.1,0.3,0.5)$.

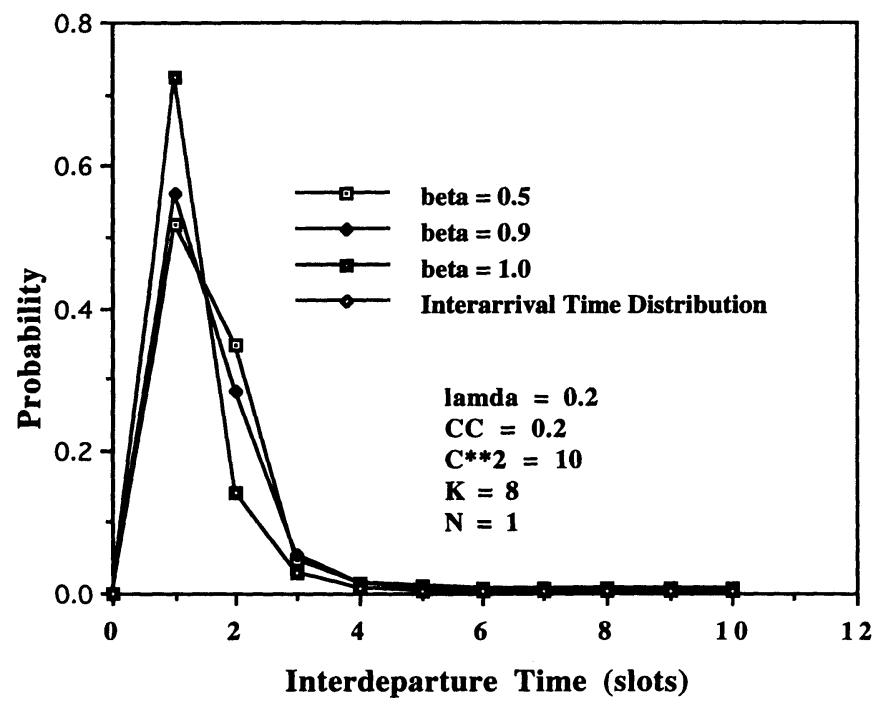

Figure 5 Interdeparture time disttribution for the MMBP-stream w.r.t. the amount of the BP stream $(\beta=0.5,0.9,1.0)$. 


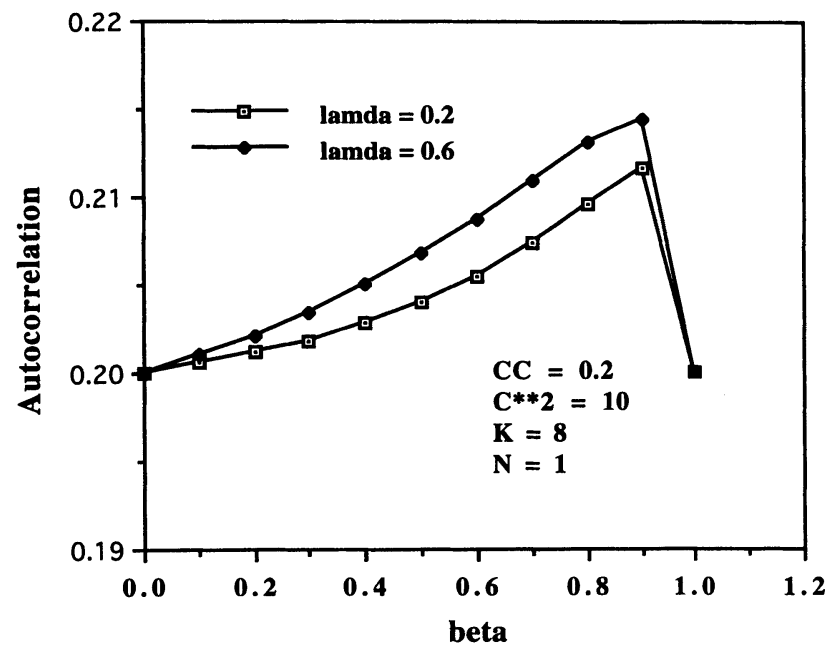

Figure 6 Autocorrelation of the interdeparture time distribution for the MMBP-stream as a function of the amount of the BP stream.

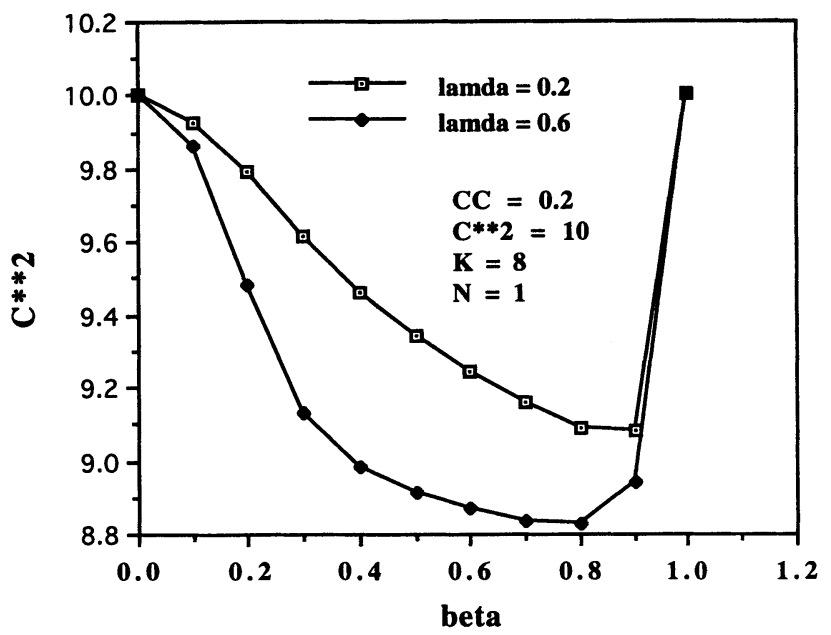

Figure $7 \mathrm{C}^{2}$ of the interdeparture time distribution for the MMBP-stream as a function of the amount of the BP stream. 


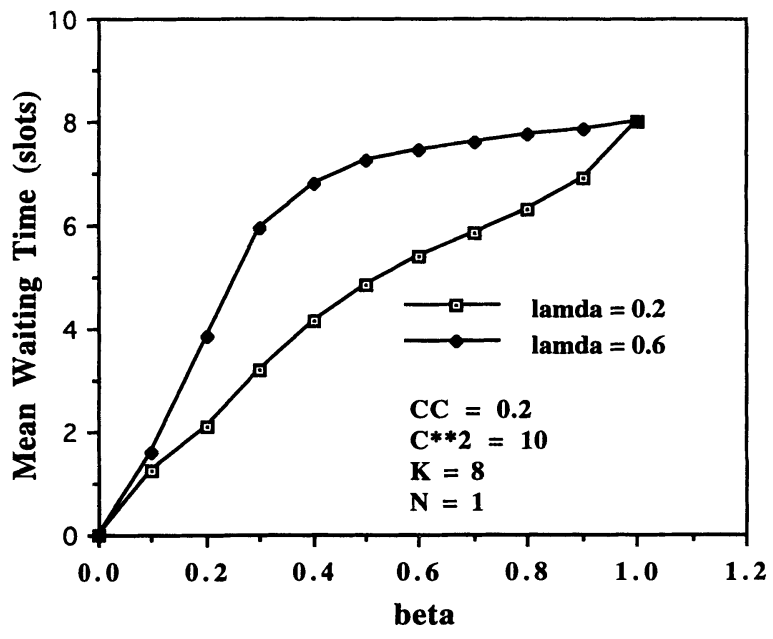

Figure 8 Mean waiting time for the MMBP-stream packets as a function of the amount of the BP stream packets.

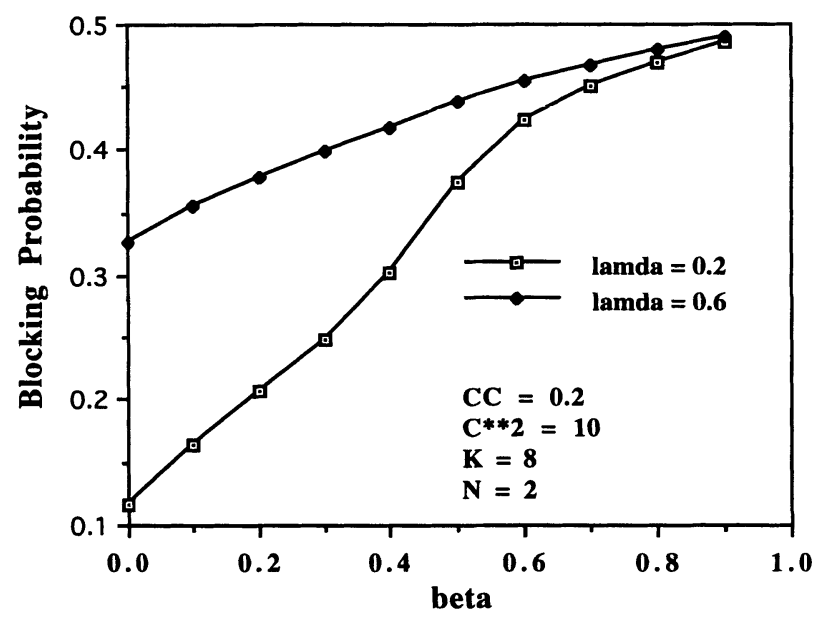

Figure 9 Blocking probability for the MMBP-stream packets as a function of the amount of the BP traffic. 


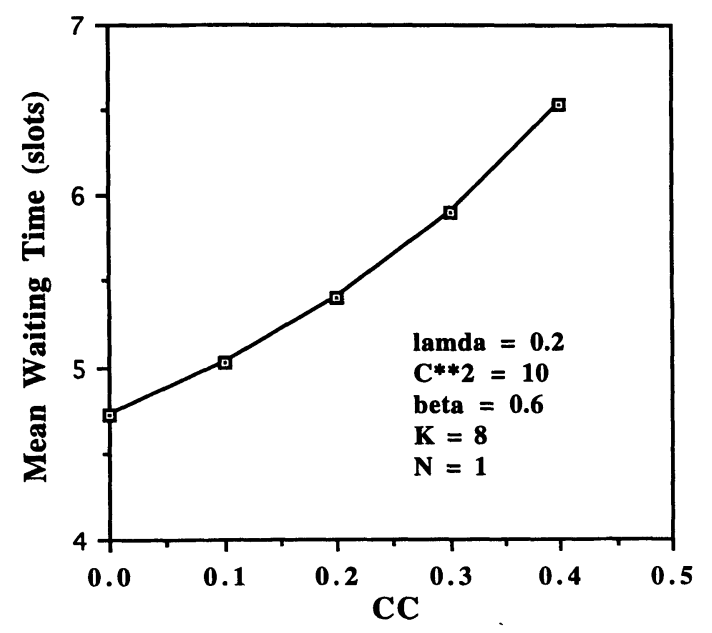

Figure 10 Mean waiting time for the MMBP-stream packets as a function of the degree of the correlation of the MMBP-stream.

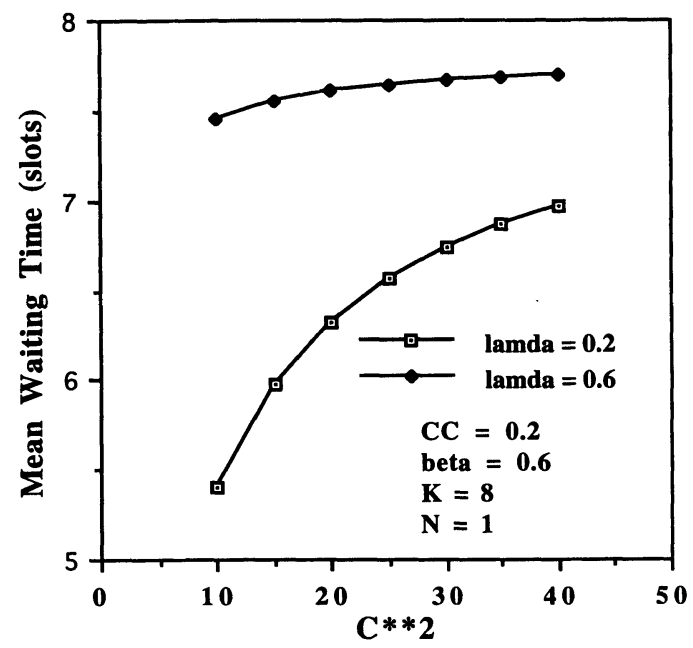

Figure 11 Mean waiting time for the MMBP-stream packets as a function of the degree of the burstiness of the MMBP-stream. 


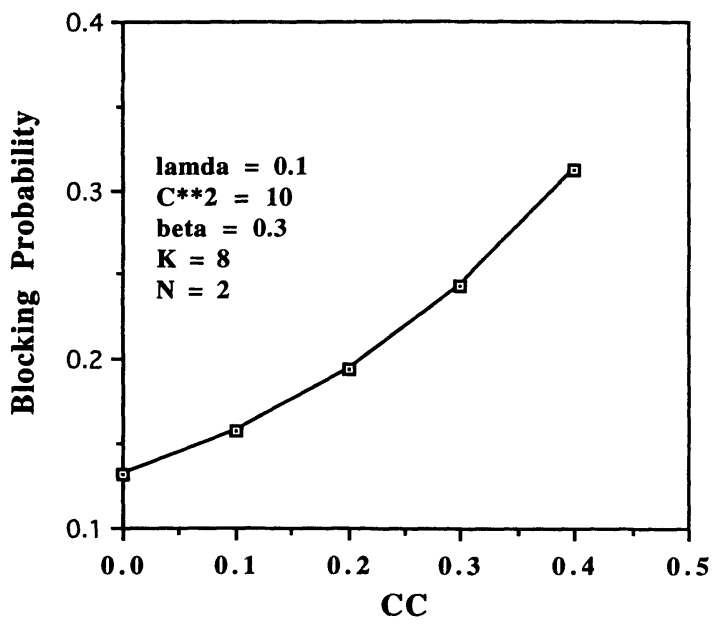

Figure 12 Blocking probability for the MMBP-stream packets as a function of the degree of the correlation of the MMBP-stream.

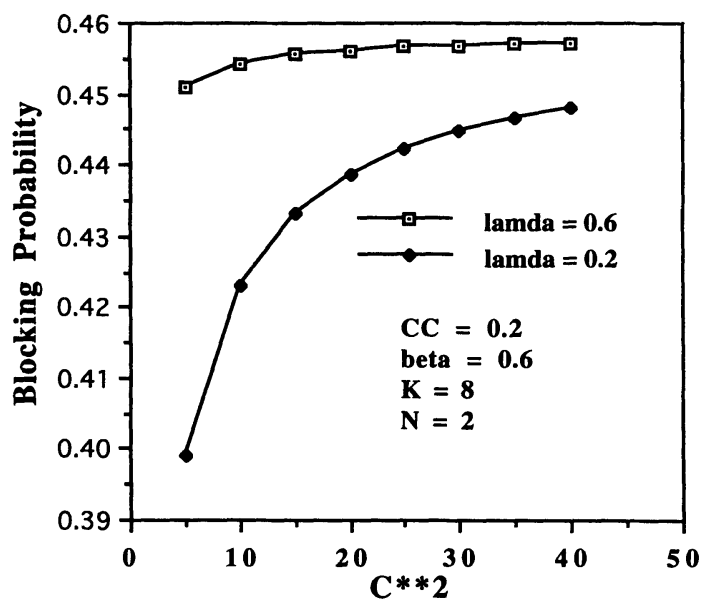

Figure 13 Blocking probability for the MMBP-stream packets as a function of the degree of the burstiness of the MMBP-stream. 


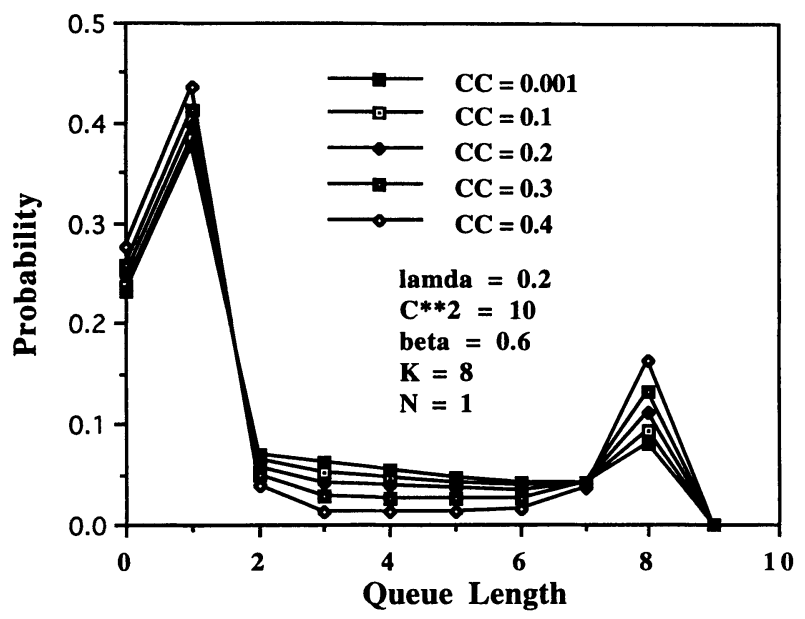

Figure 14 Queue length distribution at arbitrary points in time w.r.t. the degree of the correlation of the MMBP-stream.

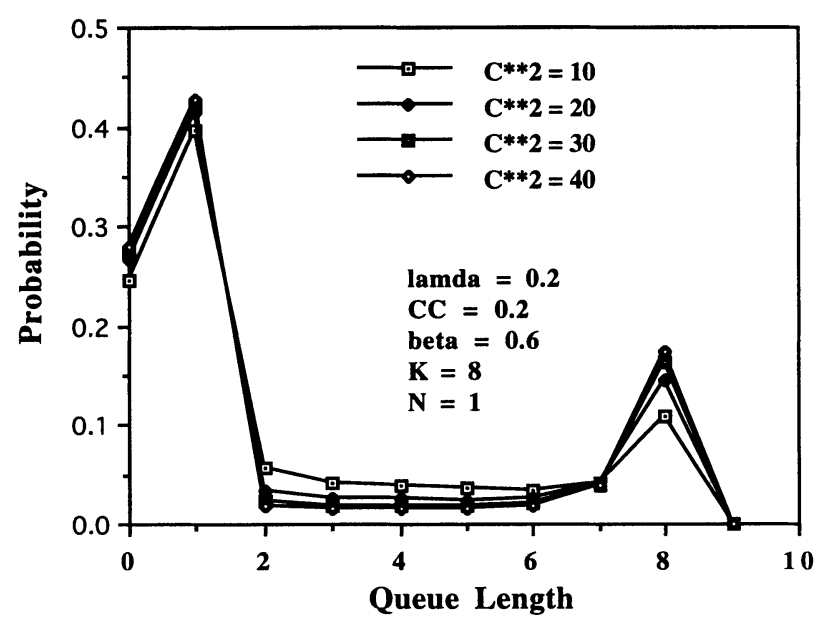

Figure 15 Queue length distribution at arbitrary points in time w.r.t. the degree of the burstiness of the MMBP-stream. 


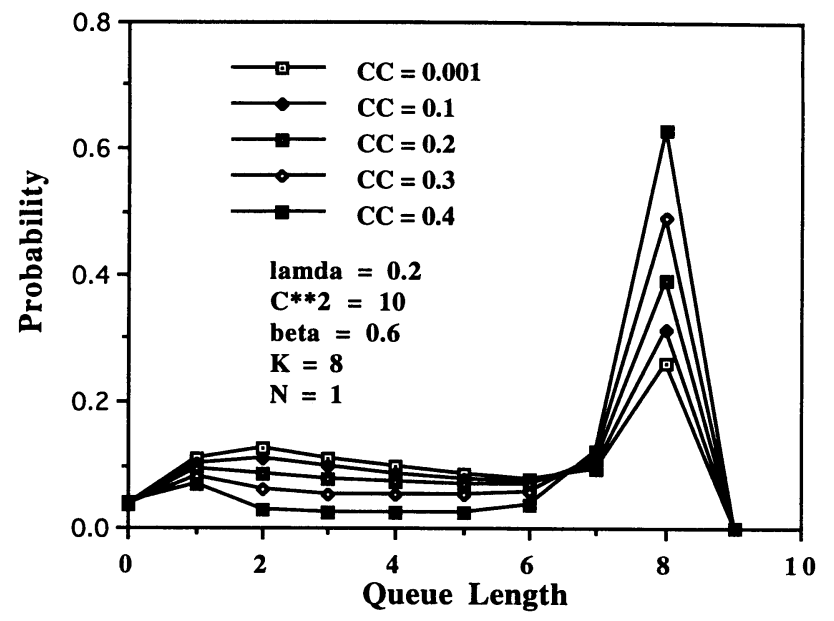

Figure 16 Queue length distribution at the MMBP-stream packet arrival instances w.r.t. the degree of the correlation of the MMBP-stream.

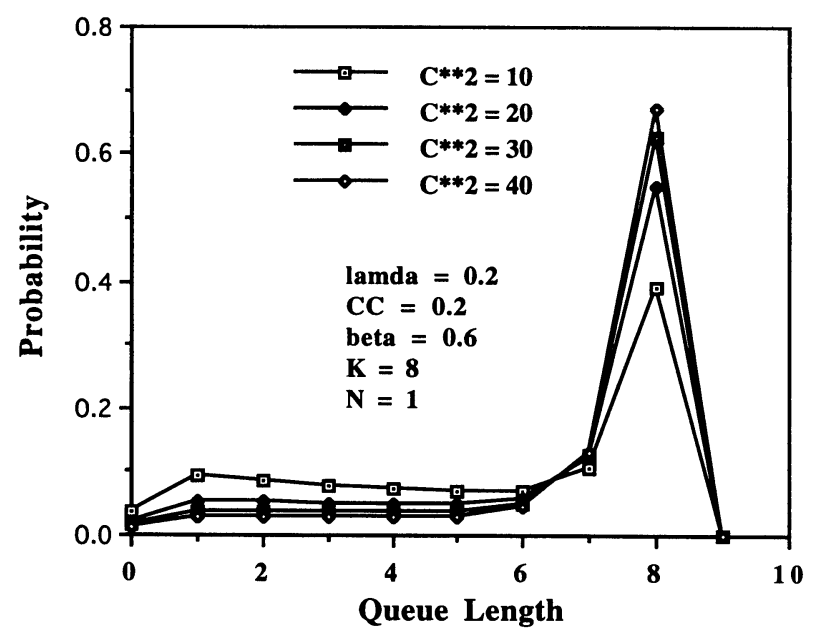

Figure 17 Queue length distribution at the MMBP-stream packet arrival instances w.r.t. the degree of the burstiness of the MMBP-stream. 